Review Article

\title{
New Hypothesis on Pathogenesis of Ovarian Cancer Lead to Future Tailored Approaches
}

\author{
P. Rescigno, I. Cerillo, R. Ruocco, C. Condello, S. De Placido, and M. Pensabene \\ Department of Clinical Medicine and Surgery, University Hospital and University Federico II, via Pansini 5, 80131 Naples, Italy \\ Correspondence should be addressed to M. Pensabene; matrod@libero.it
}

Received 18 February 2013; Accepted 14 May 2013

Academic Editor: Athanassios Dellas

Copyright (C) 2013 P. Rescigno et al. This is an open access article distributed under the Creative Commons Attribution License, which permits unrestricted use, distribution, and reproduction in any medium, provided the original work is properly cited.

\begin{abstract}
In the last decades, management of epithelial ovarian cancer (EOC) has been based on the staging system of the International Federation of Gynecology and Obstetrics (FIGO), and different classifications have been proposed for EOC that take account of grade of differentiation, histological subtype, and clinical features. However, despite taxonomic efforts, EOC appears to be not a unique disease; its subtypes differ for epidemiological and genetic risk factors, precursor lesions, patterns of spread, response to chemotherapy, and prognosis. Nevertheless, carboplatin plus paclitaxel combination represents the only standard treatment in adjuvant and advanced settings. This paper summarizes theories about the classification and origin of EOC and classical and new prognostic factors. It presents data about standard treatment and novel agents. We speculate about the possibility to create tailored therapy based on specific mutations in ovarian cancer and to personalize prevention.
\end{abstract}

\section{Introduction}

Ovarian cancer is the seventh most common cancer in women worldwide (incidence) and the 2nd leading cause of death worldwide (mortality) among gynecological malignancies. However, malignant epithelial ovarian tumors (carcinomas) are the most lethal gynecological malignancies [1]. In the last decades, management of epithelial ovarian cancer (EOC) has been based on the staging system of the International Federation of Gynecology and Obstetrics (FIGO) and the evaluation of histological features of tumors [2]. Platinum-based schedules have represented the gold standard of cure, just recently improved with the introduction of bevacizumab in front line $[3,4]$. Two studies showed better outcome particularly in progression-free survival (PFS) and a positive trend in overall survival (OS) with biological treatment $[5,6]$. The most recent hypothesis on the pathogenesis of ovarian cancer introduces the concept of a different disease, opening new frontiers and scenarios for the future treatment $[7,8]$. As for other cancers, in which the identification of specific biomarkers and biological features has led to target and tailored treatments, in future, the right treatment for the right patient could be chosen also for ovarian cancer.

Here, we reviewed the relevant clinical aspects of the theories on the pathogenesis of EOC and the potential implications by translating molecular research findings in preventive and treatment settings.

\section{Ovarian Cancer: One Name for Different Diseases}

The ovarian cancers may be distinguished, according to the grade of differentiation, from nuclear atypia and the presence or absence of stromal invasion into three groups: benign, borderline, and malignant tumors (carcinoma) [9]. The EOCs are a heterogeneous group of tumors that can be classified according to the histology in serous, mucinous, endometrioid, clear cell, and transitional and squamous tumors [9].

In order to explain the heterogeneity of EOC, Kurman and Shih proposed a new classification which divided ovarian carcinomas into two categories, type I and type II [7]. 
Type I includes low-grade serous, low-grade endometrioid, clear cell, and mucinous carcinomas. These cancers account for $25 \%$ of ovarian malignancies and cause $10 \%$ of deaths. These tumors frequently are diagnosed in early stage and have indolent behavior and good prognosis. These tumors are characterized by slow growth and appear as voluminous and unilateral masses. They are characterized by genetic stability, and frequent mutations have been described for KRAS, BRAF, PTEN, PIK3CA, and ERBB2. For type I ovarian carcinomas, morphological precursors have been identified as those leading to the development of invasive tumors by successive transformations.

Type II includes the high-grade serous, high-grade endometrioid, and undifferentiated carcinomas. They account for $75 \%$ of ovarian cancers and cause $90 \%$ of deaths. These tumors are diagnosed in advanced stage because of rapid growth [7]. They are characterized by genomic instability such as p53 mutation [10], inactivation of BRCA1/2 [11], and CCNE1 amplification.

According to Prat, the classification of ovarian cancer in just two types could be reductive from clinical point of view, so he divided EOC in five groups: high-grade serous (HGSC), endometrioid (ECs), clear cell (CCCs), mucinous (MCs), and low-grade serous (LGSC) [8]. Those variants represent distinct diseases, as supported by differences in epidemiology, genetic risk factors, molecular events, premalignant lesions, patterns of spread, response to chemotherapy, and prognosis [8].

HGSCs are the most common ovarian carcinomas (70\%) and occur in the advanced stage and spread beyond the ovary at diagnosis. They exhibit p53, BRCA,WT1, and p16 mutations and high ki67 levels and frequently express estrogen receptors (ERs). ERs are also expressed in LGSCs and ECs, but they are negative in almost all CCCs and MCs.

LGSCs account for $<5 \%$ of all cases of EOC, are frequently associated with a serous borderline tumor and follow a relatively indolent course. They show KRAS and BRAF mutations but not BRCA and p53 alterations.

MCs are 3-4\% of ovarian tumors, show gastrointestinal differentiation, large size, and unilaterality, and are usually confined to the ovary. KRAS mutations are an early event in mucinous tumorigenesis. These tumors are also frequently immunoreactive for cytokeratin 7 and 20.

ECs represent $10 \%$ of all ovarian carcinomas. They occur more frequently in perimenopausal age and at an early stage. These tumors are bilateral in $28 \%$ of cases, are associated with $15-20 \%$ of cases with endometrium carcinomas, and seem to arise from endometriotic cysts. High-grade ECs are morphologically indistinguishable from HGSCs, and they often express WT1. ECs are characterized by ARID1A mutations and CTNNB abnormalities and are associated with favorable outcome and PTEN inactivation or PIK3CA mutations; ECs are also immunoreactive for vimentin, cytokeratins 7 and 20, ERs, and progesterone receptors.

CCCs account for $10 \%$ of ovarian carcinomas, and patients typically present early stage disease. They are associated with endometriosis and show unfavorable prognosis when they are present at advanced stage. These tumors carry ARID1A mutations and are usually positive for HNF1- $\beta$ [8].

\section{Ovarian Cancer: What Origin?}

Traditional theory led back the origin of EOC to ovarian surface epithelium (mesothelium), and subsequent metaplastic changes lead to the development of the different cell types (serous, endometrioid, clear cell, mucinous, and transitional cell) [12]. Recent theory introduced the concept that the different histotypes of EOC originated from three different sites. In Figure 1, the origin of EOC from fimbria, endometrial tissue, and tubal-mesothelium junction is depicted. According to this theory, serous ovarian cancer originates from the fallopian tubes [13].

In carriers of BRCA mutation, a lesion similar to a serous tubal intraepithelial carcinoma (STIC) [14] has been described. Therefore, adnexal mass could originate from implantation of tubal tumor cells to the ovary [15]. Many studies have shown that the STIC and small invasive cancers can be recognized not only in women with a genetic predisposition but also in 50-60\% of sporadic ovarian cancers [16]. The first transformation process seems to take place in the secretory cells of tube [17]. In addition, p53 mutations have been identified in the STIC synchronous with highgrade serous carcinomas. A recent study on the genetic expression of high-grade serous carcinomas also showed a higher correlation with the tubal epithelium than with the ovarian surface epithelium [7].

Dysplastic and hyperplastic lesions were frequently found in fallopian tubes in BRCA1/2 mutation carriers that underwent prophylactic surgery. Such lesions showed changes in cell cycle and apoptosis related proteins suggesting a premalignant phenotype. Moreover, in this setting, occult carcinomas have been reported in $2-11 \%$ of the ovaries removed. The majority of these occult carcinomas are seen within the fallopian tube, especially in the fimbriae, which has led to the hypothesis that many BRCA-associated ovarian cancers may have originated in the fallopian tube $[19,20]$.

A condition was then described in which the tubal cells are apparently normal with an overexpression of p53, defined "p53 signature" [21]. This is comprised exclusively of secretory cells, and the majority exhibit evidence of DNA damage. About 57\% contain p53 mutations with a low Ki67 proliferation index. It is unclear if the $\mathrm{p} 53$ signature represents a premalignant lesion or a benign overexpression of $\mathrm{p} 53$ to DNA damages without biological relevance [7]. p53 mutation is an early event in the genesis of HGSC, occurring in p53 signature foci and leading to STIC in the distal fallopian tube. BRCA1 mutations also occur early during the development of STIC but after p53 mutation [22].

LGSCs originate from a noninvasive serous borderline tumor. They represent the progression of this precursor beyond microinvasion. The presence of small foci of LGSC and ovarian borderline tumor is associated with an excellent prognosis. Advance stage LGSCs are less favorable; nevertheless, the disease usually follows a relatively indolent course [7].

ECs and low-grade CCCs originate from endometriotic cysts and are frequently associated with implantation of endometriosis in the pelvis [22]. The origin of endometriosis has not yet been clarified. It is unclear whether it is related 


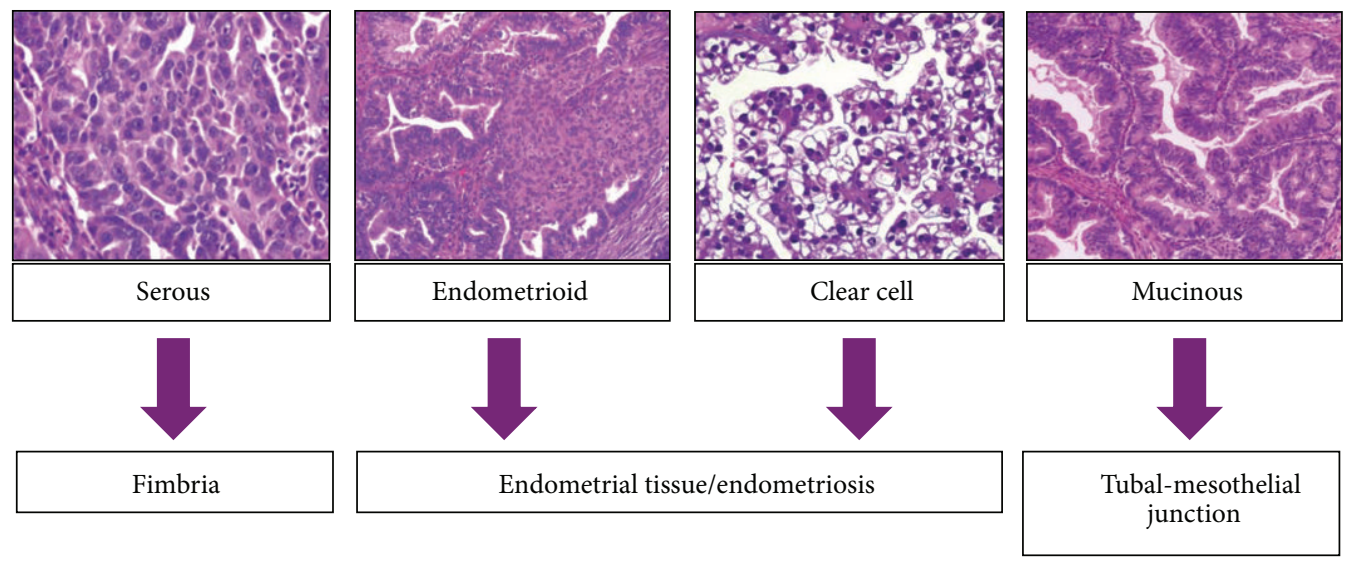

FIgure 1: Pathogenesis of different EOCs according to Prat.

to a process of metaplasia or flow retrograde menstrual. The latter hypothesis is the most reasonable suggesting that ECs and CCCs develop from the endometrial tissue implanted on the ovary. Endometriosis shows molecular abnormalities, including the activation of the oncogenetic pathway [23] that allows the endometrial tissue to implant, survive, and invade the ovarian and peritoneal tissue. The tubal ligation seems to have a protective effect on the development of endometrioid and clear cell carcinoma because this procedure prevents the retrograde menstrual flow, while it does not prevent high-grade serous carcinomas because it does not avoid the possible exfoliation of abnormal tubal cells [7].

The primary MCs of the ovary are rare, accounting for 3\% of EOC. The origin of these tumors is not clear because it is not possible to recognize a Müllerian phenotype. Although it has been suggested that they may have a similar origin to endocervical tumors, the epithelium that characterizes the MCs is more similar to the gastrointestinal mucosa. An association between MCs and tumors of Brenner (transitional cell) has been described. They show the "Walthard cell nest's" elements consisting of a benign transitional epithelium, which have been found in the paraovarian and paratubal cysts. Therefore, the MCs may originate from transitional cell at the tube-peritoneal junction [7].

\section{Prognostic and/or Predictive Factors Are Not a Shared Consensus}

Histologic subtype, tumor grade, and disease stage are currently used to stratify patients into high versus low-risk disease and predict response to therapy. These features allow the clinicians to divide early EOCs in two risk classes: low risk (stage IA-B, G1-2) and high risk (stages IA-B, G3, and IC-II, clear cell or undifferentiated histology) [24]. However, EOC is diagnosed at an early stage (I and II FIGO stages) only in about $25 \%$ of the cases; more frequently ( $70 \%$ of cases), the diagnosis is posed at late stages. The five-year survival of these patients is $70-90 \%$ in stage I, $50-60 \%$ in stage II, $20-40 \%$ in stage III, and $10 \%$ in stage IV [25].

A large series study, conducted on 575 women affected by EOC, optimally surgically debulked without macroscopic residual disease, showed that tumor cell type was both more reproducible and provided superior prognostic information compared with assignment of tumor grade $[26,27]$. Tumorcell type was confirmed to be the most relevant histopathological prognostic also in patients treated with surgery and chemotherapy [28]. MCs and CCCs have been associated with inferior outcome [29-31].

Significance of tumor grade remained only for low-versus high-grade serous carcinomas, while grade 2 and 3 tumors had a similar prognosis. Tumor grade prognostic significance is irrelevant in MCs, ECs, and CCCs subtypes [32].

EOCs appear as a heterogeneous disease. DNA or cellular alterations that lead tumor behaviour, not routinely detected, should represent new prognostic or predictive factors.

The cell cycle is regulated by two major families of cyclin kinase inhibitors (CKIs). p21 and p27 are members of Cip/kip family and inhibit cyclin E/CDK [33] arresting cells in G1 phase.

Wild type p53 is a negative regulator of cell cycle progression and works on activating p21 expression. It also induces cells to apoptosis when DNA damages are revealed [34]. Tumor protein 53 (p53) alterations are present in 96\% of HGSCs but are rare in LGSCs [35].

Mutations of the p21 and p27 genes occur less frequently in human cancer than p53 alterations [36]. Immunostaining (IHC) of p21 alone [37], or concomitant loss of p21 (negativity), overexpression of p53 (positivity) [38], and the combination of low p21 and p27 expression were associated with worse overall survival [39] for EOC in several studies. In a recent study, p53 and p21 status in 129 ovarian cancers was not related to serous/nonserous tumors, tumor grade, or FIGO stage. However, the p53 status was significantly associated with disease-free survival (DFS) in a univariate analysis. Patients with p53 negative tumors had a 5-year DFS of $82 \%$ compared with $62 \%$ in p53 positive tumors [40].

The role of HER2 in EOC is still unclear. HER2 overexpression is associated with poor prognosis, and ovarian cancers are estimated to be HER2 positive in $10 \%$ of cases [41]. Up-to-date there is no consensus about the evaluation protocol applied for HER2 overexpression in EOC. New tools for the detection of HER2 in ovarian cancer have been evaluated 
such as real-time polymerase chain reaction (PCR) analysis on cancer cells captured by laser microdissection in order to optimize the identification of patients overexpressing HER2 that could benefit from a target therapy with trastuzumab or pertuzumab [42].

Amplicon-dependent expression of the cell cycle protein cyclin E (CCNE1) has been recently detected as predictor of survival in advanced EOC [43]. CCNE1 amplification represents an oncogenic stimulus through the activation of the cell cycle and contributes to primary resistance to cisplatin. Moreover, CCNE1 gene amplification is also a predictive biomarker of therapeutic response for several new targeted therapeutic options, as BMS-387032, P1446A-05, flavopiridol, and seliciclib [36].

BRCA1 and BRCA2 mutations, caused by germline or somatic alterations, play a key role in ovarian carcinogenesis. The BRCA genes encode proteins that enter in mismatch repair systems, particularly homologous recombination, repairing DNA double helix damage [44]. BRCA1 or BRCA2 mutations are a high-risk factor for HGSC, and women harboring such mutations have a $30 \%$ to $70 \%$ probability of developing ovarian cancer by the age of 70 $[45,46]$.

Incidence of germline or somatic mutations in BRCA1/2 genes is up to $22 \%$ in HGSC. BRCA1/2 function can be lost also through DNA hypermethylation or epigenetic silencing (BRCAness phenotype), but these mechanisms are mutually exclusive of either BRCA1 or BRCA2 mutations [36]. Defects in homologous-recombination repair can also be caused by loss of function of proteins other than BRCA1 and BRCA2, including the RAD51, ataxia telangiectasia mutated (ATM), ataxia telangiectasia and Rad3 related (ATR), and checkpoint kinase 1 and 2 homologue (CHK1 and CHK2) proteins, as well as components of the Fanconi's anemia repair pathway [44]. Loss of function of these proteins also sensitizes cells to PARP inhibition. Such defects in homologous-recombination repair may be relatively common in some sporadic ovarian cancers [47], potentially making this therapeutic strategy more widely useful as an anticancer treatment. Only one wild allele of BRCA1 or BRCA2 is sufficient for DNA repair mechanism, so as in Knudson's model, and additional somatic loss of the wild-type allele is necessary to develop ovarian carcinoma in women with a germline BRCA mutation [48]. Furthermore, when BRCA is mutated, homologous recombination does not work and DNA damage cannot be repaired. Carboplatin acts just for creating DNA double helix damage, which justifies BRCA mutant patients better prognosis compared to sporadic forms [49].

TP53 mutations and ovarian cancers with loss of BRCA1 and/or BRCA2 function seem to be target for new therapies as inhibitors of poly(ADP-ribose) polymerase (PARP). So that detecting BRCA mutations should be predictor of therapy response [50].

K-RAS is a member of family able to regulate cell growth, survival, and differentiation activating downstream effectors [51]. Mutations in KRAS gene are typical of lowgrade ovarian tumors while are rare in III and IV stages [52]. Other mechanisms able to deregulate KRAS gene in ovarian cancer are gene amplifications that account in about $11 \%$ of ovarian tumors [53] and short RNA molecule (miRNA) let-7 [54]. Let-7 binds to its specific site in the $3^{\prime}$-UTR of KRAS mRNA and induces KRAS downregulation [54]. Recently, KRAS-LCS6 polymorphism has shown to be not relevant in ovarian cancer and not associated to any outcome or physiopathological characteristic [55].

Mutations in the p110 subunit of PI3K, called PIK3CA, are often responsible for activation of the phosphatidylinositol 3-kinase (PI3K)/AKT/mammalian target of rapamycin (mTOR) pathway and have been detected in $12 \%$ of ovarian cancers [56]. Several preclinical studies suggested that PIK3CA mutations could predict response to PI3K and mTOR inhibitors, although mutations in the mitogenactivated protein kinase (MAPK) pathway (KRAS, NRAS, BRAF) might mediate resistance $[57,58]$.

In a recent study, twenty-three PIK3CA-mutant women, after a first-line standard therapy, were treated with an inhibitor of this pathway showing a higher response rate $(30 \%)$ compared to patients under standard treatment (10\%) independently from their primary malignancies, that is, breast or gynaecological cancers [59].

Mutations in BRAF and KRAS, components of the mitogen-activated protein kinase (MAPK) cascade, are common in LGSCs and serous borderline ovarian tumors, but they are present in $<1 \%$ of HGSCs. So that patients with BRAF mutations may have an improved clinical outcome [60].

However, serous borderline and LGSCs commonly are chemotherapy-resistant diseases, with response rates to chemotherapy of $4 \%$ in the neoadjuvant setting and $2.1 \%-$ $4.9 \%$ in the recurrent setting [61].

Given the high prevalence of BRAF and KRAS mutations in these tumors, there has been recent interest in testing inhibitors targeting the MAPK pathway in patients with advanced disease. Nevertheless, patients with aggressive LGSCs do not harbour BRAF mutation typically. Moreover, vemurafenib, a selective RAF inhibitor, lacks his activity in KRAS mutant and BRAF/RASWT tumors, so it could have a limited utility in this setting of patients [62].

On the contrary, a recent phase 2 trial of the MEK (MAP kinase kinase) inhibitor AZD6244 in women with recurrent LGSCs of the ovary or peritoneum reported a radiographic response rate of $15.4 \%$ [63].

A recent trial has shed new light on this regard. It demonstrated that BRAF mutations are common in surgically treated patients who did not recur but are rarely present in patients who required systemic therapy. It suggested that highly selective RAF inhibitors may have limited utility in this disease; nevertheless, a subset of LGSCs depends on MEK activity [64].

ARID1A is a mutated gene in a significant portion of CCCs and ECs, but it is not detected in serous ovarian tumors [18]. The ARID1A gene encodes a member of the SWI/SNF family, BAF250a, that is able to regulate gene transcription altering chromatin structure [18]. It is detectable in atypical endometriosis, suggesting that this is an early event in a multistep carcinogenesis of ECs. Recently, loss of BAF250a was reported in almost half of ECS and CCCs, but no association was found between BAF250a expression and any clinical and pathological risk factors or survival. 
EOCs are associated with high levels of vascular endothelial growth factor (VEGF), a protein related to tumor growth and metastatic process. VEGF is correlated with worse outcome of EOCs patients [65].

\section{Conventional Treatments of EOC}

The therapy for stage I is undoubtedly total abdominal hysterectomy and bilateral salpingo-oophorectomy with careful surgical staging. Pelvic and periaortic nodes may be involved in $10-20 \%$ of the time in apparent stage I disease, and lymphadenectomy is considered an important diagnostic and therapeutic procedure [3].

Adjuvant chemotherapy is indicated in early stages except for low risk (stage IA-B, G1-2) EOCs. ICON 1 trial showed an increase in PFS and OS in patients who received platinumbased adjuvant treatment, especially for the high-risk group (G3 IA, IB, IC G2-G3, clear cell) [66].

The combination of carboplatin and paclitaxel has represented the first-line standard treatment more for early than for advanced EOC, as demonstrated by GOG 111 [67] and OV 10 trials [68]. Just recently, the association of bevacizumab has gained better PFS. Among the most promising targets identified in ovarian cancer, a leading role belongs to angiogenesis. In recent years, several phase II studies have demonstrated the toxicity profile and activity of bevacizumab, a monoclonal recombinant antibody that binds VEGF-A, in the treatment of relapsed ovarian cancer $[69,70]$. Two recent phase III trials, that is, the GOG218 $[5,6]$ and ICON7, have shown the efficacy of bevacizumab associated to standard treatment, in prolonging PFS of about four months compared to chemotherapy alone (10.7 versus 14.1 months). A preliminary analysis has indicated a trend in improving OS. These findings led the European Medicines Agency (EMA) to the approval of bevacizumab in combination with carboplatin and paclitaxel as upfront treatment in the in advanced EOCs, peritoneal cancers, and tubal cancers.

Despite the availability of a fist-line single treatment, the different subtypes of EOCs showed dissimilar outcome to chemotherapy. CCCs and MCs are less likely to respond to chemotherapy, but particularly CCCs show a lower 5year survival in late stage than HGSCs (20\% versus 30\%) $[71,72]$. Indeed, whereas highly proliferative cells of HGSC show sensitivity to platinum due to loss of the ability to repair double-stranded DNA, the less proliferative CCCs are less sensitive to platinum compounds because of their more genetically stable cells [73].

Recent studies demonstrated greater sensitivity to platinum based chemotherapy for patients with p53 mutation, in contrast to previously believed [74].

As well as p53 mutations, BRCA deficient cells show a higher sensitivity to platinum compound in preclinical setting [75]. In clinical setting, Bolton et al. have shown an improved 5-year OS in BRCA1 and BRCA2-related EOCs at a standard treatment, despite a later stage and higher grade at diagnosis. The 5 -year OS was $36 \%$ for noncarriers, $44 \%$ for BRCA1 carriers, and 52\% for BRCA2 carriers. After adjusting for study and year of diagnosis, BRCA1 and BRCA2 mutation carriers showed a more favourable survival than noncarriers. These survival differences remained after additional adjustment for stage, grade, histology, and age at diagnosis. BRCA2 carriers had the best prognosis [76].

\section{Novel Agents}

Novel agents are under investigation in EOCs. In particular, genetic mutations can be used as molecular targets for new selective pharmacological agents.

Different chemotherapeutic agents, such as liposomal doxorubicin and trabectedin, are observed to have higher response rates in patients with BRCA mutation or with BRCAness phenotype [77].

In this setting, also PARP inhibitors have been studied. PARPs are multifunctional enzymes that play an important role in the repair of DNA single-strand breaks. The inhibition of PARPs causes the accumulation of DNA single-strand breaks leading to DNA double-strand breaks. Normal cells are able to repair this damage by homologous recombination, but in BRCA1/2 mutation carriers, these lesions are not repaired resulting in cell cycle arrest and cell death. Farmer et al. demonstrated that the decreasing PARP1 expression with RNA expression interference resulted in the reduction of the survival of BRCA1/2 embryonic stem cells compared with wild-type cells [78]. The first-in-human clinical trial of the PARP inhibitor, olaparib, has been conducted in patients with BRCA1/2-mutated advanced cancers including ovarian, breast, and prostate cancers [79]. Olaparib demonstrated a very acceptable side effect profile when compared with conventional chemotherapies. A durable antitumor activity was found in cancer associated with the BRCA1 or BRCA2 mutation. These data indicate that using PARP inhibition to target a specific DNA-repair pathway has the necessary selectivity profile and a wide therapeutic window for $B R C A$-deficient cells, supporting the clinical relevance of the hypothesis that $B R C A$ mutation-associated cancers are susceptible to a synthetic lethal therapeutic approach [80, 81].

In the ICEBERG2 study, Audeh et al. provided positive proof of the concept of the efficacy and tolerability of genetically targeted treatment with olaparib in BRCAmutated advanced ovarian cancer [82]. Activity of olaparib was observed both in platinum-sensitive and platinumresistant patients, suggesting that resistance mechanisms to olaparib might only partly overlap with those for platinum chemotherapies. Although phase III trials in EOC are not available, in triple negative breast cancer, a negative phase III trial opened the concept of a better understanding of mechanisms supporting PARP resistance and a better definition of clinical setting for PARP inhibitors development [83].

A recent analysis in patients with BRCA1/2-mutated ovarian cancer has suggested that chemosensibility, particularly with carboplatin and taxanes, is maintained in such patients after disease progression on olaparib [84].

Kristjansdottir and Dizon have recently reviewed the role of HER-dimerization inhibitors, in particular evaluating the scientific rationale of pertuzumab in ovarian cancers. To date, pertuzumab is the most extensively studied HER2 inhibitor in ovarian cancer, with almost 400 patients having been evaluated in phase II studies. Pertuzumab showed limited 
TABLE 1: The correlation of the five types of Prat theory and clinical implications.

\begin{tabular}{|c|c|c|c|c|c|}
\hline & HGSH & LGSH & MC & EC & CCC \\
\hline \multicolumn{6}{|l|}{ Prat theory } \\
\hline Precursor lesions & STIC & $\begin{array}{l}\text { Serous borderline } \\
\text { tumor }\end{array}$ & $\begin{array}{l}\text { Cystoadenoma/borderline } \\
\text { tumor }\end{array}$ & $\begin{array}{l}\text { Atypical } \\
\text { endometriosis }\end{array}$ & $\begin{array}{l}\text { Atypical } \\
\text { endometriosis }\end{array}$ \\
\hline Pattern of spread & $\begin{array}{l}\text { Very early } \\
\text { transcoelomic spread }\end{array}$ & $\begin{array}{l}\text { Transcoelomic } \\
\text { spread }\end{array}$ & Usually limited to ovary & $\begin{array}{l}\text { Usually limited to } \\
\text { pelvis }\end{array}$ & $\begin{array}{l}\text { Usually limited to } \\
\text { pelvis }\end{array}$ \\
\hline Molecular & BRCA & BRAF & KRAS & PTEN & HNF1 \\
\hline abnormalities & P53 & KRAS & HER2 & ARID1A & ARID1A \\
\hline Chemosensitivity & High & Intermediate & Low & High & Low \\
\hline Prognosis & Poor & Intermediate & Favorable & Favorable & Intermediate \\
\hline \multicolumn{6}{|l|}{ Clinical implications } \\
\hline $\begin{array}{l}\text { Potential } \\
\text { target therapies }\end{array}$ & PARP inhibitors & $\begin{array}{l}\text { BRAF } \\
\text { KRAS inhibitors }\end{array}$ & $\begin{array}{l}\text { Monoclonal antibodies } \\
\text { Anti-HER2 } \\
\text { KRAS inhibitors }\end{array}$ & Not Available & Not available \\
\hline $\begin{array}{l}\text { Potential role of } \\
\text { screening }\end{array}$ & $\begin{array}{l}\text { Research of novel } \\
\text { early biomarkers }\end{array}$ & $\begin{array}{l}\text { Research of novel } \\
\text { early biomarkers }\end{array}$ & $\begin{array}{l}\text { Transvaginal ultrasound }+ \\
\text { CA125 }\end{array}$ & $\begin{array}{l}\text { Transvaginal } \\
\text { ultrasound + } \\
\text { CA125 }\end{array}$ & $\begin{array}{l}\text { Transvaginal } \\
\text { ultrasound + } \\
\text { CA125 }\end{array}$ \\
\hline
\end{tabular}

HGSC: high-grade serous carcinoma; LGSC: low-grade serous carcinoma; EC: endometrioid carcinoma; CCC: clear cell carcinoma; MC: mucinous carcinoma; STIC: serous tubal intraepithelial carcinoma.

activity in platinum-sensitive and platinum-resistant disease in recurrent ovarian cancer when combined with chemotherapy. However, a subset analysis suggests that pertuzumab has a high response in the subgroup of patients with activated HER2 or low HER3 mRNA expression. Therefore, HER2 activation and HER3 mRNA levels may predict response to pertuzumab in ovarian cancer [85]. Further prospective biomarker-led trials are warranted.

A large phase II study concluded that the clinical value of single-agent trastuzumab in recurrent ovarian cancer is limited by the low frequency of HER2 overexpression and low rate of objective response among patients with HER2 overexpression [86].

The negative findings of the previous studies can be explained by the hypothesis that other molecular pathways interfere with the EGFR/HER2 signalling, such as follicle stimulating hormone receptor (FSHR), potentially minimizing HER2 impact on ovarian cancer cell proliferation and ultimately to the HER2 effect on disease progression and prognosis. HER2 can be a negative prognosticator only in FSHR negative EOC cases [87].

\section{Implications for Screening, Prevention, and Treatment}

Early detection of EOC has represented a challenge in the last decades. Pelvic examination, transvaginal ultrasound, and detection of serum CA125 are current measures in cancer screening, without reaching evidence of good outcome [24]. The dualistic model of the pathogenesis of EOC suggests the need of different approaches for prevention of different types of EOCs. Type I tumors (low-grade serous, low-grade endometrioid, clear cell, and mucinous) are slow growing and reach a large size while still confined to the ovary [7]. They could be detected by pelvic examination and/or transvaginal ultrasound. The early diagnosis of type II EOC is a more complex challenge. Type II tumors (high-grade serous, undifferentiated carcinomas, and malignant mixed mesodermal tumors) originate outside the ovary [7] and suggest new screening approaches, by developing a panel of sensitive and specific biomarkers that are expressed early in ovarian carcinogenesis.

Prophylactic salpingo-oophorectomy is currently recommended as strategy to reduce ovarian cancer risk [88] in BRCA1 and BRCA2 mutation carriers. As in the PROSE consortium study [89], prophylactic surgery significantly reduces ovarian cancer $(\mathrm{HR}=0.31 ; 95 \% \mathrm{CI}=0.12-0.82)$ and breast cancer risk when performed in women under 50 years, especially in BRCA2 mutation carriers $(\mathrm{HR}=0.36$; $95 \% \mathrm{CI}=$ $0.16-0.82)$ than in BRCA1 ( $\mathrm{HR}=0.63 ; 95 \% \mathrm{CI}=0.41-0.96)$. At a median followup of about 5 years, breast $(\mathrm{HR}=0.44$; $95 \% \mathrm{CI}=0.26-0.76)$ and ovarian cancer specific $(\mathrm{HR}=0.25$; $95 \% \mathrm{CI}=0.08-0.75)$ mortality rates were reduced. Premature or precocious menopause has been associated with longterm effects [90], that is, increased cardiovascular disease, cognitive impairment, psychiatric symptoms, impaired sexual function, osteoporosis, and bone fractures. The recent hypothesis on the pathogenesis of ovarian cancer BRCAcorrelated from fimbria and the subsequent implants on the surface of the ovaries could modify prophylactic strategies limiting the surgery to tubes, sparing the endocrine function of the ovaries, and avoiding the short- and long-term effects on women's health of such risk management option.

As with early detection, the treatment of type I and type II tumors must be individualized. Type I tumors are generally low grade, slow growing, and localized to the ovary at diagnosis, spreading late in their evolution. Accordingly, when confined to the ovary, conservative surgery, that is, salpingooophorectomy, could be a sufficient approach. On the other hand, when an adjuvant treatment is required according to 


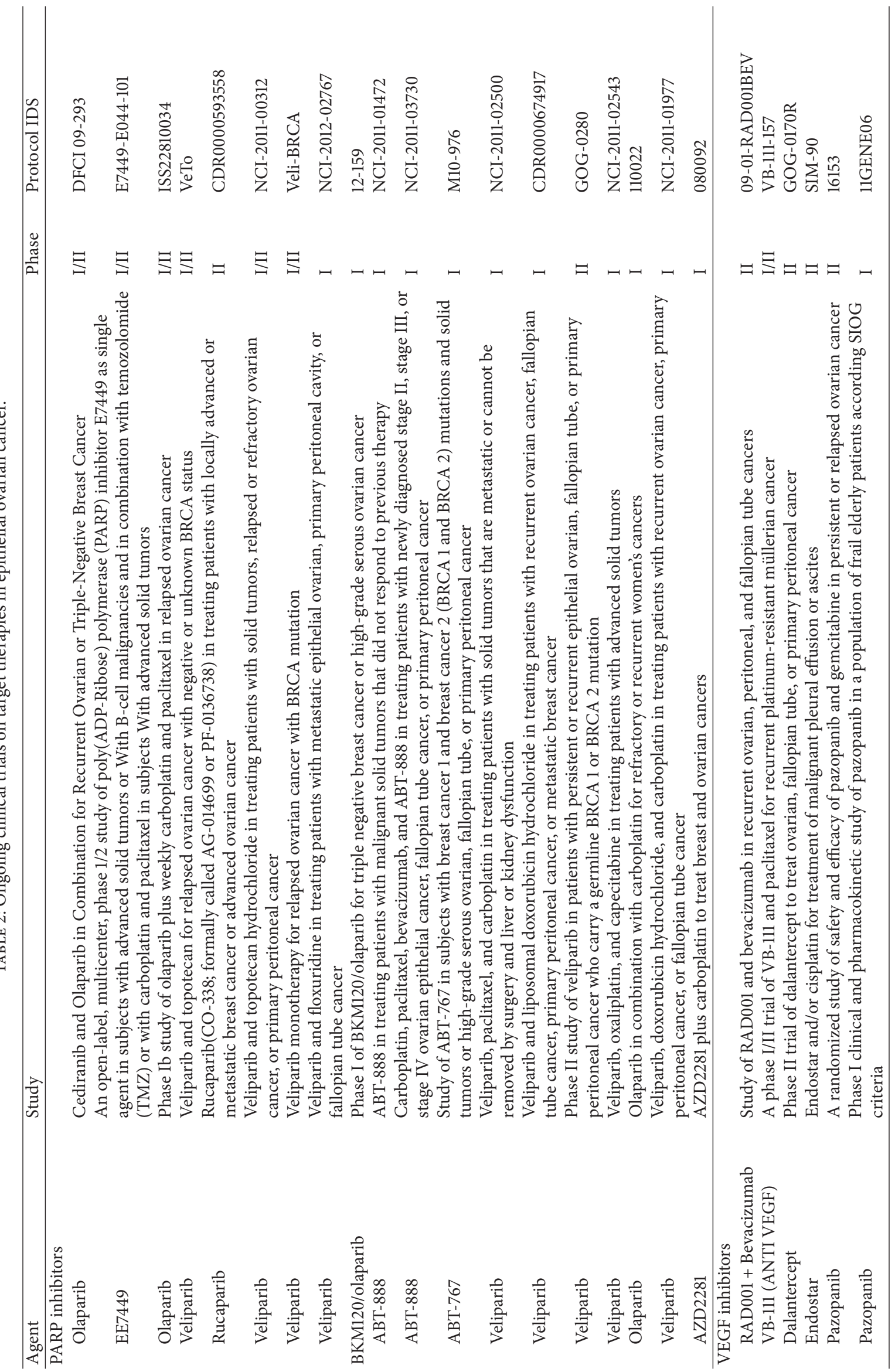




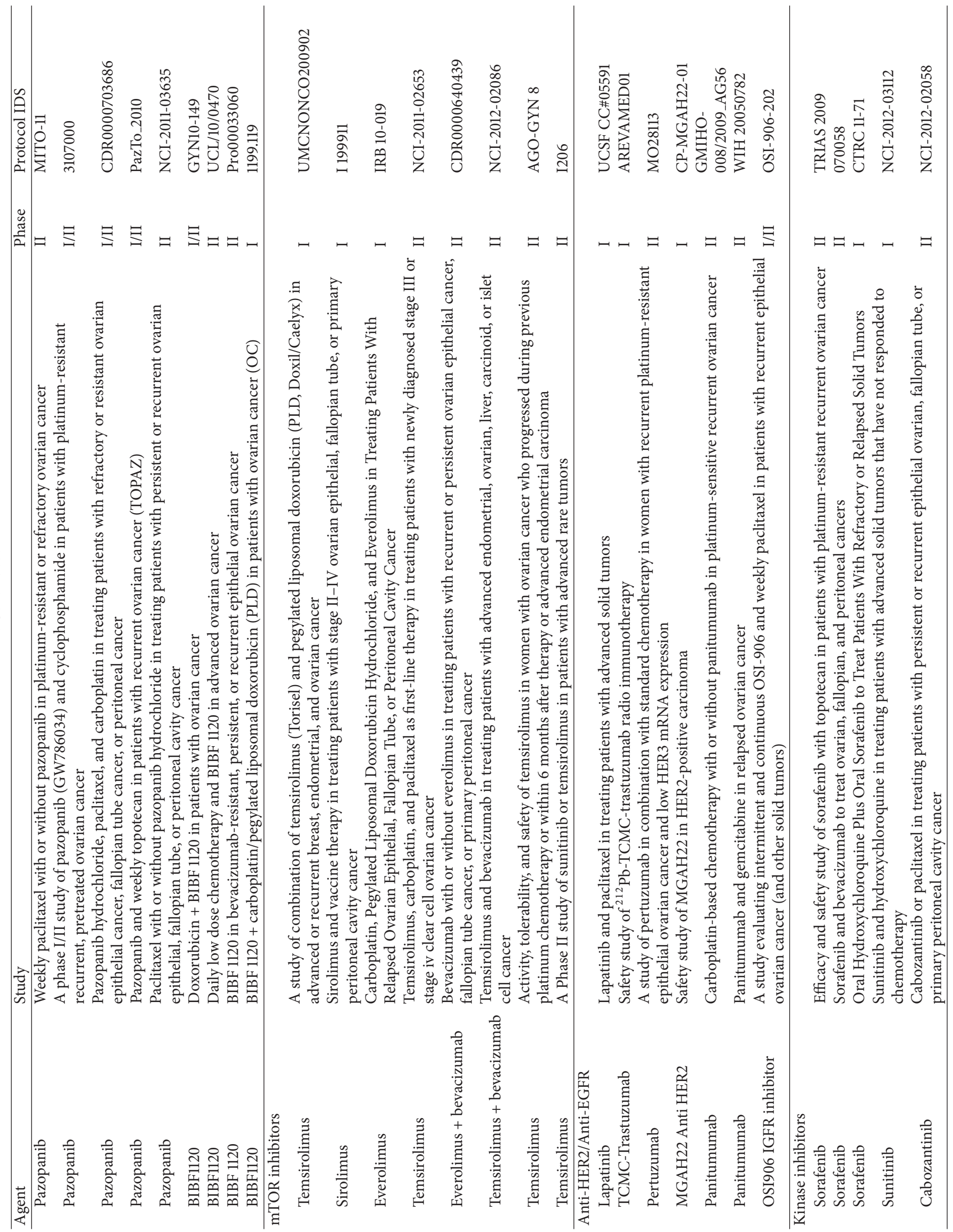




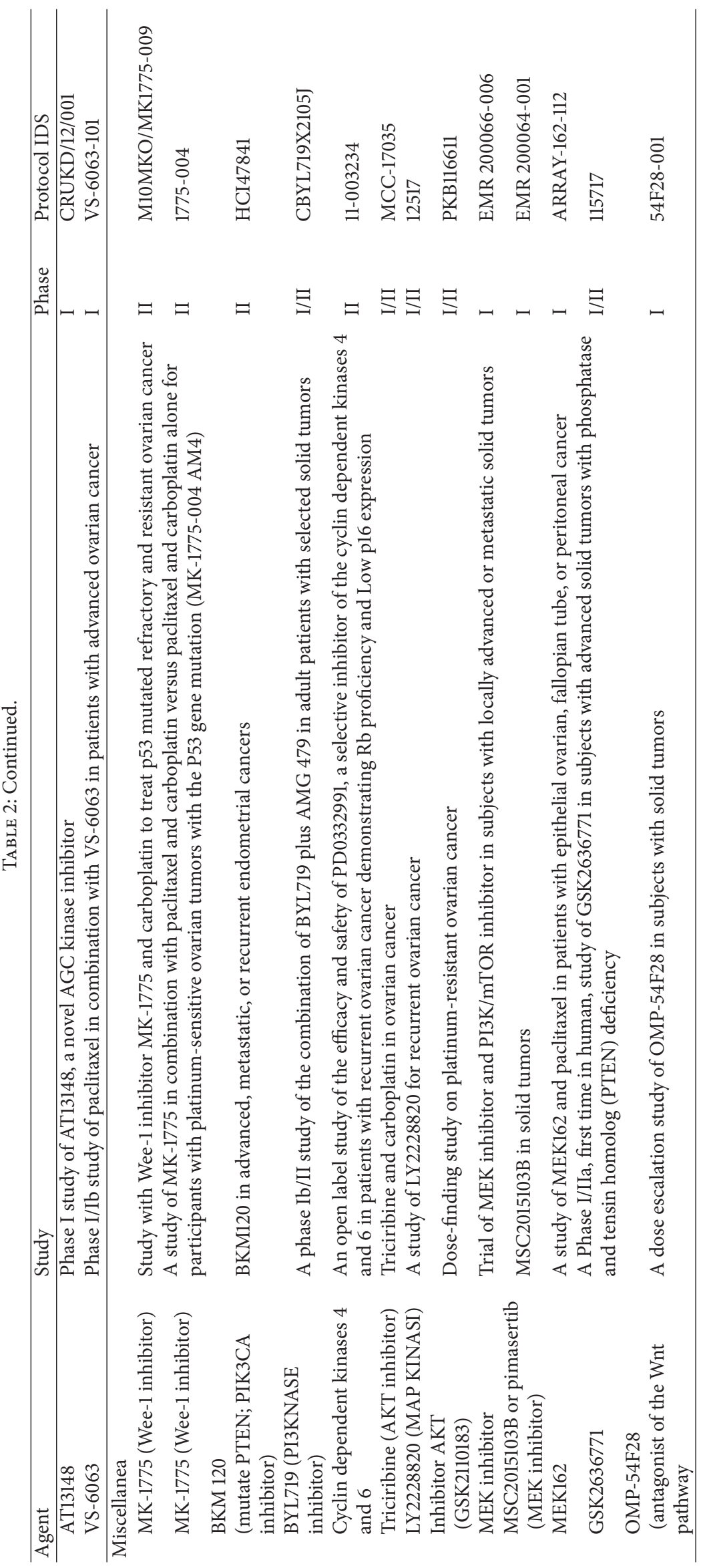


stage, different schedules could be improved for type I and type II tumors, in agreement of the peculiar biomolecular profile. In type I EOC, it is reasonable to develop in clinical setting BRAF inhibitors and other MAPK kinase inhibitors in order to improve progression-free survival and overall survival in patients with advanced EOC [62], actually treated with conventional chemotherapeutic schedules.

In Table 1, Prat theory has been summarized with a focus on the clinical implications about the potential drugs to be used for the five different subtypes of EOC.

On the other hand, type II EOC, currently identified as EOC with a germline or acquired mutations in BRCA genes, as well as sporadic ovarian cancer with a BRCAness phenotype [91], could benefit the recognition of molecular mechanisms involved in PARP pathway. The potential benefit of PARP inhibitors might be maximized in type II ovarian cancer. It will be crucial to explore novel therapeutic trial strategies and drug combinations, including PARP inhibitors, and incorporate robust biomarkers predictive of response if these drugs are to reach their full potential.

In Table 2, ongoing clinical trials on target therapies in EOC are summarized. Trials have been selected among studies registered at the canver.gov website [92].

\section{Conclusion}

Regardless of dualistic or five subtypes hypothesis, the molecular characterization of ovarian cancer in different subtypes is the cornerstone to build a personalized approach in the era of personalized medicine.

\section{Conflict of Interests}

The authors declare that they have no conflict of interests.

\section{References}

[1] GLOBOCAN 2008 v1.2. Cancer Incidence and Mortality Worldwide, IARC CancerBase, no. 10, IARC Press, Lyon, France, 2010, http://globocan.iarc.fr/.

[2] A. P. Heintz, F. Odicino, P. Maisonneuve et al., "Carcinoma of the ovary. FIGO 26th annual report on the results of treatment in gynecological cancer," International Journal of Gynaecology and Obstetrics, vol. 95, supplement 1, pp. S161-S192, 2006.

[3] N. Colombo, D. Guthrie, S. Chiari et al., "International Collaborative Ovarian Neoplasm trial 1: a randomized trial of adjuvant chemotherapy in women with early-stage ovarian cancer," Journal of the National Cancer Institute, vol. 95, no. 2, pp. 125-132, 2003.

[4] M. J. Piccart, K. Bertelsen, K. James et al., "Randomized intergroup trial of cisplatin-paclitaxel versus cisplatincyclophosphamide in women with advanced epithelial ovarian cancer: three-year results," Journal of the National Cancer Institute, vol. 92, no. 9, pp. 699-708, 2000.

[5] R. A. Burger, M. F. Brady, M. A. Bookman et al., "Incorporation of bevacizumab in the primary treatment of ovarian cancer," The New England Journal of Medicine, vol. 365, pp. 2473-2483, 2011.

[6] T. J. Perren, A. M. Swart, J. Pfisterer et al., "A phase 3 trial of bevacizumab in ovarian cancer," The New England Journal of Medicine, vol. 365, no. 26, pp. 2484-2496, 2011.
[7] R. J. Kurman and I.-M. Shih, "Molecular pathogenesis and extraovarian origin of epithelial ovarian cancer-shifting the paradigm," Human Pathology, vol. 42, no. 7, pp. 918-931, 2011.

[8] J. Prat, "Ovarian carcinomas: five distinct diseases with different origins, genetic alterations, and clinicopathological features," Virchows Archiv, vol. 460, no. 3, pp. 237-249, 2012.

[9] K. R. Lee, F. A. Tavassoli, J. Prat et al., "Tumours of the ovary and peritoneum: surface epithelial-stromal tumours," in World Health Organization Classification of Tumours. Pathology and Genetics of Tumours of the Breast and Female Genital Organs, F. A. Tavassoli and P. Devilee, Eds., chapter 2, pp. 117-145, IARC Press, Lyon, France, 2003.

[10] A. A. Ahmed, D. Etemadmoghadam, J. Temple et al., "Driver mutations in TP53 are ubiquitous in high grade serous carcinoma of the ovary," Journal of Pathology, vol. 221, no. 1, pp. 49$56,2010$.

[11] E. Senturk, S. Cohen, P. R. Dottino, and J. A. Martignetti, "A critical re-appraisal of BRCA1 methylation studies in ovarian cancer," Gynecologic Oncology, vol. 119, no. 2, pp. 376-383, 2010.

[12] R. J. Kurman and I.-M. Shih, "The origin and pathogenesis of epithelial ovarian cancer: a proposed unifying theory," American Journal of Surgical Pathology, vol. 34, no. 3, pp. 433443, 2010.

[13] J. M. J. Piek, P. J. van Diest, R. P. Zweemer et al., "Dysplastic changes in prophylactically removed Fallopian tubes of women predisposed to developing ovarian cancer," Journal of Pathology, vol. 195, no. 4, pp. 451-456, 2001.

[14] G. Chêne, F. Penault-Llorca, G. le Bouëdec et al., "Ovarian epithelial dysplasia and prophylactic oophorectomy for genetic risk," International Journal of Gynecological Cancer, vol. 19, no. 1, pp. 65-72, 2009.

[15] J. M. J. Piek, R. H. M. Verheijen, P. Kenemans, L. F. Massuger, H. Bulten, and P. J. van Diest, "BRCA1/2-related ovarian cancers are of tubal origin: a hypothesis," Gynecologic Oncology, vol. 90, no. 2, p. 491, 2003.

[16] M. J. Callahan, C. P. Crum, F. Medeiros et al., "Primary fallopian tube malignancies in BRCA-positive women undergoing surgery for ovarian cancer risk reduction," Journal of Clinical Oncology, vol. 25, no. 25, pp. 3985-3990, 2007.

[17] D. W. Kindelberger, Y. Lee, A. Miron et al., "Intraepithelial carcinoma of the fimbria and pelvic serous carcinoma: evidence for a causal relationship," American Journal of Surgical Pathology, vol. 31, no. 2, pp. 161-169, 2007.

[18] K. C. Wiegand, S. P. Shah, O. M. Al-Agha et al., "ARID1A mutations in endometriosis-associated ovarian carcinomas," The New England Journal of Medicine, vol. 363, no. 16, pp. 1532$1543,2010$.

[19] M. L. Carcangiu, P. Radice, S. Manoukian et al., "Atypical epithelial proliferation in fallopian tubes in prophylactic salpingo-oophorectomy specimens from BRCA1 and BRCA2 germline mutation carriers," International Journal of Gynecological Pathology, vol. 23, no. 1, pp. 35-40, 2004.

[20] F. Medeiros, M. G. Muto, Y. Lee et al., "The tubal fimbria is a preferred site for early adenocarcinoma in women with familial ovarian cancer syndrome," American Journal of Surgical Pathology, vol. 30, no. 2, pp. 230-236, 2006.

[21] Y. Lee, A. Miron, R. Drapkin et al., "A candidate precursor to serous carcinoma that originates in the distal fallopian tube," The Journal of Pathology, vol. 211, no. 1, pp. 26-35, 2007.

[22] E. Veras, T.-L. Mao, A. Ayhan et al., "Cystic and adenofibromatous clear cell carcinomas of the ovary: distinctive tumors that 
differ in their pathogenesis and behavior: a clinicopathologic analysis of 122 cases," American Journal of Surgical Pathology, vol. 33, no. 6, pp. 844-853, 2009.

[23] S. E. Bulun, "Endometriosis," The New England Journal of Medicine, vol. 360, no. 3, pp. 268-279, 2009.

[24] B. T. Hennessy, R. L. Coleman, and M. Markman, "Ovarian cancer," The Lancet, vol. 374, no. 9698, pp. 1371-1382, 2009.

[25] "FIGO annual report, vol. 24," The Journal of Epidemiology and Biostatistics, vol. 6, pp. 107-138, 2001.

[26] C. B. Gilks, D. N. Ionescu, S. E. Kalloger et al., "Tumor cell type can be reproducibly diagnosed and is of independent prognostic significance in patients with maximally debulked ovarian carcinoma," Human Pathology, vol. 39, no. 8, pp. 12391251, 2008.

[27] A. Bamias, M. Sotiropoulou, F. Zagouri et al., "Prognostic evaluation of tumour type and other histopathological characteristics in advanced epithelial ovarian cancer, treated with surgery and paclitaxel/carboplatin chemotherapy: cell type is the most useful prognostic factor," European Journal of Cancer, vol. 48, no. 10, pp. 1476-1483, 2012.

[28] M. Köbel, S. E. Kalloger, P. M. Baker et al., "Diagnosis of ovarian carcinoma cell type is highly reproducible: a transcanadian study," American Journal of Surgical Pathology, vol. 34, no. 7, pp. 984-993, 2010.

[29] R. J. Zaino, M. F. Brady, S. M. Lele, H. Michael, B. Greer, and M. A. Bookman, "Advanced stage mucinous adenocarcinoma of the ovary is both rare and highly lethal," Cancer, vol. 117, no. 3, pp. 554-562, 2011.

[30] J. Alexandre, I. Ray-Coquard, F. Selle et al., "Mucinous advanced epithelial ovarian carcinoma: clinical presentation and sensitivity to platinum-paclitaxel-based chemotherapy, the GINECO experience," Annals of Oncology, vol. 21, no. 12, pp. 2377-2381, 2010.

[31] R. J. Zaino, M. F. Brady, S. M. Lele, H. Michael, B. Greer, and M. A. Bookman, "Advanced stage mucinous adenocarcinoma of the ovary is both rare and highly lethal: a Gynecologic Oncology Group study," Cancer, vol. 117, no. 3, pp. 554-562, 2011.

[32] A. Ayhan, R. J. Kurman, A. Yemelyanova et al., "Defining the cut point between low-grade and high-grade ovarian serous carcinomas: a clinicopathologic and molecular genetic analysis," American Journal of Surgical Pathology, vol. 33, no. 8, pp. 12201224, 2009.

[33] C. le Page, D. G. Huntsman, D. M. Provencher, and A.-M. Mes-Masson, "Predictive and prognostic protein biomarkers in epithelial ovarian cancer: recommendation for future studies," Cancers, vol. 2, no. 2, pp. 913-954, 2010.

[34] K. Milde-Langosch and S. Riethdorf, "Role of cell-cycle regulatory proteins in gynecological cancer," Journal of Cellular Physiology, vol. 196, no. 2, pp. 224-244, 2003.

[35] J. A. Green, E. M. Berns, C. Coens et al., "Alterations in the p53 pathway and prognosis in advanced ovarian cancer: a multifactorial analysis of the EORTC Gynaecological Cancer group (study 55865)," European Journal of Cancer, vol. 42, no. 15, pp. 2539-2548, 2006.

[36] Cancer Genome Atlas Research Network, "Integrated genomic analyses of ovarian carcinoma," Nature, vol. 474, pp. 609-615, 2011.

[37] A. Bali, P. M. O’Brien, L. S. Edwards, R. L. Sutherland, N. F. Hacker, and S. M. Henshall, "Cyclin D1, p53, and p21Waf1/Cip1 expression is predictive of poor clinical outcome in serous epithelial ovarian cancer," Clinical Cancer Research, vol. 10, no. 15, pp. 5168-5177, 2004.
[38] H. E. Geisler, J. P. Geisler, G. A. Miller et al., "p21 and p53 in ovarian carcinoma: their combined staining is more valuable than either alone," Cancer, vol. 92, no. 4, pp. 781-786, 2001.

[39] A. Schmider-Ross, O. Pirsig, E. Gottschalk, C. Denkert, W. Lichtenegger, and A. Reles, "Cyclin-dependant kinase inhibitors CIP1 (p21) and KIP1 (p27) in ovarian cancer," Journal of Cancer Research and Clinical Oncology, vol. 132, no. 3, pp. 163-170, 2006.

[40] I. Skirnisdottir and T. Seidal, "Association of p21, p21 p27 and p21 p53 status to histological subtypes and prognosis in low-stage epithelial ovarian cancer," Cancer Genomic and Proteomics, vol. 10, pp. 27-34, 2013.

[41] E. Verri, P. Guglielmini, M. Puntoni et al., "HER2/neu oncoprotein overexpression in epithelial ovarian cancer: evaluation of its prevalence and prognostic significance: clinical study," Oncology, vol. 68, no. 2-3, pp. 154-161, 2005.

[42] T. Hillig, J. Thode, M. F. Breinholt et al., "Assessing HER2 amplification by IHC, FISH, and real-time polymerase chain reaction analysis (real-time PCR) following LCM in formalinfixed paraffin embedded tissue from 40 women with ovarian cancer," APMIS, vol. 120, pp. 1000-1007, 2012.

[43] N. Nakayama, K. Nakayama, Y. Shamima et al., "Gene amplification CCNE1 is related to poor survival and potential therapeutic target in ovarian cancer," Cancer, vol. 116, no. 11, pp. 26212634, 2010.

[44] A. R. Venkitaraman, "Cancer susceptibility and the functions of BRCA1 and BRCA2," Cell, vol. 108, no. 2, pp. 171-182, 2002.

[45] S. Chen and G. Parmigiani, "Meta-analysis of BRCA1 and BRCA2 penetrance," Journal of Clinical Oncology, vol. 25, no. 11, pp. 1329-1333, 2007.

[46] A. Antoniou, P. D. P. Pharoah, S. Narod et al., "Average risks of breast and ovarian cancer associated with BRCA1 or BRCA2 mutations detected in case series unselected for family history: a combined analysis of 22 studies," American Journal of Human Genetics, vol. 72, no. 5, pp. 1117-1130, 2003.

[47] J. Z. Press, A. de Luca, N. Boyd et al., "Ovarian carcinomas with genetic and epigenetic BRCA1 loss have distinct molecular abnormalities," BMC Cancer, vol. 8, article 17, 2008.

[48] B. M. Norquist, R. L. Garcia, K. H. Allison et al., "The molecular pathogenesis of hereditary ovarian carcinoma," Cancer, vol. 116, no. 22, pp. 5261-5271, 2010.

[49] Z. Wang, F. Wang, T. Tang, and C. Guo, "The role of PARP1 in DNA damage response and its application in tumor therapy," Frontiers of Medicine, vol. 6, no. 2, pp. 156-164, 2012.

[50] M. K. Weil and A. P. Chen, "PARP inhibitor treatment in ovarian and breast cancer," Current Problems in Cancer, vol. 35, no. 1, pp. 7-50, 2011.

[51] S. Schubbert, K. Shannon, and G. Bollag, "Hyperactive Ras in developmental disorders and cancer," Nature Reviews Cancer, vol. 7, no. 4, pp. 295-308, 2007.

[52] I. Vereczkey, O. Serester, J. Dobos et al., "Molecular characterization of 103 ovarian serous and mucinous tumors," Pathology and Oncology Research, vol. 17, no. 3, pp. 551-559, 2011.

[53] A. J. Hanrahan, N. Schultz, M. L. Westfal et al., "Genomic complexity and AKT dependence in serous ovarian cancer," Cancer Discovery, vol. 2, pp. 56-67, 2012.

[54] S. M. Johnson, H. Grosshans, J. Shingara et al., "RAS is regulated by the let-7 microRNA family," Cell, vol. 120, no. 5, pp. 635-647, 2005.

[55] E. Caiola, E. Rulli, R. Fruscio, A. Buda, M. Broggini, and M. Marabese, "KRas-LCS6 polymorphism does not impact on outcomes in ovarian cancer," American Journal of Cancer Research, vol. 2, no. 3, pp. 298-308, 2012. 
[56] D. A. Levine, F. Bogomolniy, C. J. Yee et al., "Frequent mutation of the PIK3CA gene in ovarian and breast cancers," Clinical Cancer Research, vol. 11, no. 8, pp. 2875-2878, 2005.

[57] N. T. Ihle, R. Lemos Jr., P. Wipf et al., "Mutations in the phosphatidylinositol-3-kinase pathway predict for antitumor activity of the inhibitor PX-866 whereas oncogenic ras is a dominant predictor for resistance," Cancer Research, vol. 69, no. 1, pp. 143-150, 2009.

[58] F. Di Nicolantonio, S. Arena, J. Tabernero et al., "Deregulation of the PI3K and KRAS signaling pathways in human cancer cells determines their response to everolimus," Journal of Clinical Investigation, vol. 120, no. 8, pp. 2858-2866, 2010.

[59] F. Janku, J. J. Wheler, S. N. Westin et al., "PI3K/AKT/mTOR inhibitors in patients with breast and gynecologic malignancies harboring PIK3CA mutations," Journal of Clinical Oncology, vol. 30, no. 8, pp. 777-782, 2012.

[60] K.-K. Wong, Y. T. M. Tsang, M. T. Deavers et al., "BRAF mutation is rare in advanced-stage low-grade ovarian serous carcinomas," American Journal of Pathology, vol. 177, no. 4, pp. 1611-1617, 2010.

[61] D. M. Gershenson, C. C. Sun, D. Bodurka et al., "Recurrent lowgrade serous ovarian carcinoma is relatively chemoresistant," Gynecologic Oncology, vol. 114, no. 1, pp. 48-52, 2009.

[62] E. W. Joseph, C. A. Pratilas, P. I. Poulikakos et al., "The RAF inhibitor PLX4032 inhibits ERK signaling and tumor cell proliferation in a V600E BRAF-selective manner," Proceedings of the National Academy of Sciences of the United States of America, vol. 107, no. 33, pp. 14903-14908, 2010.

[63] J. Farley, W. E. Brady, V. Vathipadiekal et al., "Selumetinib in women with recurrent low-grade serous carcinoma of the ovary or peritoneum: an open-label, single-arm, phase 2 study," The Lancet Oncology, vol. 14, no. 2, pp. 134-140, 2013.

[64] R. N. Grisham, G. Iyer, K. Garg et al., "BRAF mutation is associated with early stage disease and improved outcome in patients with low-grade serous ovarian cancer," Cancer, vol. 119, no. 3, pp. 548-554, 2013.

[65] D. Cheng, B. Liang, and Y. Li, "Serum vascular endothelial growth factor (VEGF-C) as a diagnostic and prognostic marker in patients with ovarian cancer," PLoS One, vol. 8, no. 2, Article ID e55309, 2013.

[66] J. B. Trimbos, M. Parmar, I. Vergote et al., "International Collaborative Ovarian Neoplasm trial 1 and Adjuvant ChemoTherapy in Ovarian Neoplasm trial: two parallel randomized phase III trials of adjuvant chemotherapy in patients with early-stage ovarian carcinoma," Journal of the National Cancer Institute, vol. 95, no. 2, pp. 105-112, 2003.

[67] W. P. McGuire, W. J. Hoskins, M. F. Brady et al., "Cyclophosphamide and cisplatin compared with paclitaxel and cisplatin in patients with stage III and stage IV ovarian cancer," The New England Journal of Medicine, vol. 334, pp. 1-6, 1996.

[68] F. M. Muggia, P. S. Braly, M. F. Brady et al., "Phase III randomized study of cisplatin versus paclitaxel versus cisplatin and paclitaxel in patients with suboptimal stage III or IV ovarian cancer: a Gynecologic Oncology Group study," Journal of Clinical Oncology, vol. 18, no. 1, pp. 106-115, 2000.

[69] L. M. Ellis and D. J. Hicklin, "VEGF-targeted therapy: mechanisms of anti-tumour activity," Nature Reviews Cancer, vol. 8, no. 8, pp. 579-591, 2008.

[70] M. R. Raspollini, F. Castiglione, F. Garbini et al., "Correlation of epidermal growth factor receptor expression with tumor microdensity vessels and with vascular endothelial growth factor expression in ovarian carcinoma," International Journal of Surgical Pathology, vol. 13, no. 2, pp. 135-142, 2005.

[71] M. Takano, Y. Kikuchi, N. Yaegashi et al., "Clear cell carcinoma of the ovary: a retrospective multicentre experience of 254 patients with complete surgical staging," British Journal of Cancer, vol. 94, no. 10, pp. 1369-1374, 2006.

[72] A. du Bois, H. J. Luck, W. Meier et al., "A randomized cinic trial of cisplatin/paclitaxel versus carboplatin/paclitaxel as first-line treatment of ovarian cancer," The Journal of the National Cancer Institute, vol. 95, pp. 1320-1329, 2003.

[73] M. Köbel, S. E. Kalloger, J. Carrick et al., "A limited Panel of immunomarkers can reliably distinguish between clear cell and high-grade serous carcinoma of theovary," American Journal of Surgical Pathology, vol. 33, no. 1, pp. 14-21, 2009.

[74] J. A. Lederman, C. Marth, M. S. Carey et al., "Role of molecular agents and targeted therapy in clinical trials for women with ovarian cancer," International Journal of Gynecological Cancer, vol. 21, no. 4, pp. 763-770, 2011.

[75] W. D. Foulkes, "BRCA1 and BRCA2: chemosensitivity, treatment outcomes and prognosis," Familial Cancer, vol. 5, no. 2, pp. 135-142, 2006.

[76] K. L. Bolton, G. Chenevix-Trench, P. D. Pharoah et al., "Association between BRCA1 and BRCA2 mutations and survival in women with invasive epithelial ovarian cancer," The Journal of the American Medical Association, vol. 307, no. 4, pp. 382-390, 2012.

[77] D. Yang, S. Khan, Y. Sun et al., "Association of BRCA1 and BRCA2 mutations with survival, chemotherapy sensitivity, and gene mutator phenotype in patients with ovarian cancer," The Journal of the American Medical Association, vol. 306, no. 14, pp. 1557-1565, 2011.

[78] H. Farmer, H. McCabe, C. J. Lord et al., "Targeting the DNA repair defect in BRCA mutant cells as a therapeutic strategy," Nature, vol. 434, no. 7035, pp. 917-921, 2005.

[79] P. C. Fong, D. S. Boss, T. A. Yap et al., "Inhibition of poly(ADPribose) polymerase in tumors from BRCA mutation carriers," The New England Journal of Medicine, vol. 361, no. 2, pp. 123134, 2009.

[80] A. Ashworth, "A synthetic lethal therapeutic approach: poly(ADP) ribose polymerase inhibitors for the treatment of cancers deficient in DNA double-strand break repair," Journal of Clinical Oncology, vol. 26, no. 22, pp. 3785-3790, 2008.

[81] W. G. Kaelin Jr., "The concept of synthetic lethality in the context of anticancer therapy," Nature Reviews Cancer, vol. 5, no. 9, pp. 689-698, 2005.

[82] M. W. Audeh, J. Carmichael, R. T. Penson et al., "Oral poly(ADP-ribose) polymerase inhibitor olaparib in patients with BRCA1 or BRCA2 mutations and recurrent ovarian cancer: a proof-of-concept trial," The Lancet, vol. 376, no. 9737, pp. 245251, 2010.

[83] T. A. Yap, S. K. Sandhu, C. P. Carden, and J. S. de Bono, "Poly (ADP-ribose) polymerase (PARP) inhibitors: exploiting a synthetic lethal strategy in the clinic," CA: A Cancer Journal for Clinicians, vol. 61, no. 1, pp. 31-49, 2011.

[84] J. Ang, T. A. Yap, P. C. Fong et al., "Preliminary experience with the use of chemotherapy (CT) following treatment with olaparib, a poly(ADP-ribose) polymerase inhibitor (PARPi), in patients with BRCA1/2-deficient ovarian cancer (BDOC)," Journal of Clinical Oncology, vol. 28, no. 15, supplement, 2010, abstract no. 5041 . 
[85] K. Kristjansdottir and D. Dizon, "HER-dimerization inhibitors: evaluating pertuzumab in women's cancers," Expert Opinion on Biological Therapy, vol. 10, no. 2, pp. 243-250, 2010.

[86] M. A. Bookman, K. M. Darcy, D. Clarke-Pearson, R. A. Boothby, and I. R. Horowitz, "Evaluation of monoclonal humanized anti-HER2 antibody, trastuzumab, in patients with recurrent or refractory ovarian or primary peritoneal carcinoma with overexpression of HER2: a phase II trial of the Gynecologic Oncology Group," Journal of Clinical Oncology, vol. 21, no. 2, pp. 283-290, 2003.

[87] S. Heublein, T. Vrekoussis, D. Mayr et al., "Her-2/neu expression is a negative prognosticator in ovarian cancer cases that do not express the follicle stimulating hormone receptor (FSHR)," Journal of Ovarian Research, vol. 6, no. 1, article 6, 2013.

[88] G. F. Schwartz, K. S. Hughes, H. T. Lynch et al., "Proceedings of the international consensus conference on breast cancer risk, genetics, \& risk management, April, 2007," Cancer, vol. 113, no. 10, pp. 2627-2637, 2008.

[89] S. M. Domchek, T. M. Friebel, C. F. Singer et al., "Association of risk-reducing surgery in BRCA1 or BRCA2 mutation carriers with cancer risk and mortality," The Journal of the American Medical Association, vol. 304, no. 9, pp. 967-975, 2010.

[90] L. T. Shuster, B. S. Gostout, B. R. Grossardt, and W. A. Rocca, "Prophylactic oophorectomy in premenopausal women and long-term health," Menopause International, vol. 14, no. 3, pp. 111-116, 2008.

[91] N. Turner, A. Tutt, and A. Ashworth, "Hallmarks of 'BRCAness' in sporadic cancers," Nature Reviews Cancer, vol. 4, no. 10, pp. 814-819, 2004.

[92] February 2013, http://www.cancer.gov/clinicaltrials. 


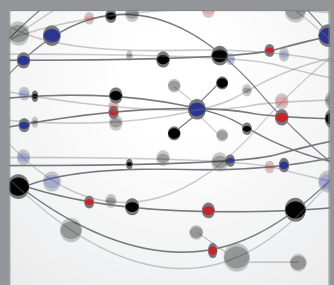

The Scientific World Journal
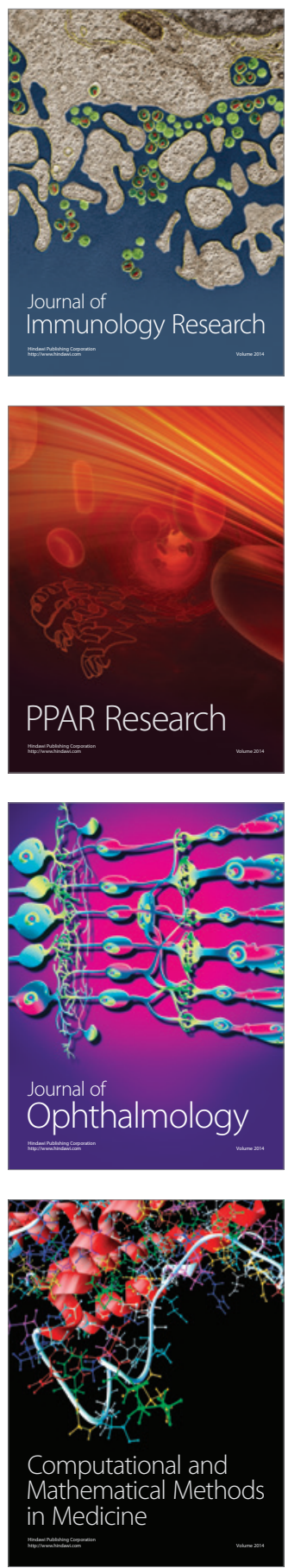

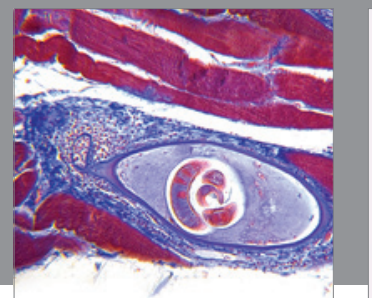

Gastroenterology

Research and Practice
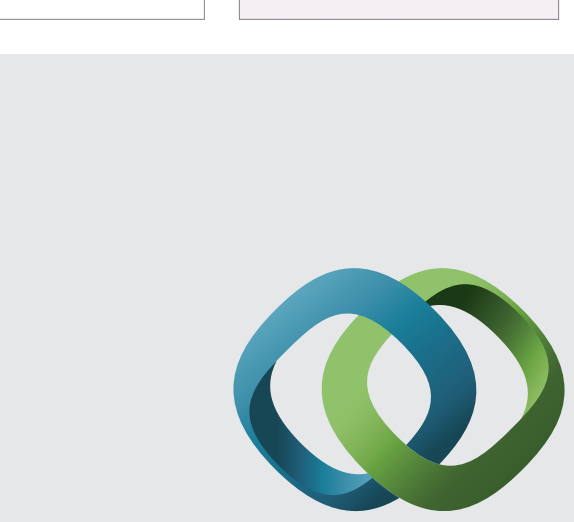

\section{Hindawi}

Submit your manuscripts at

http://www.hindawi.com
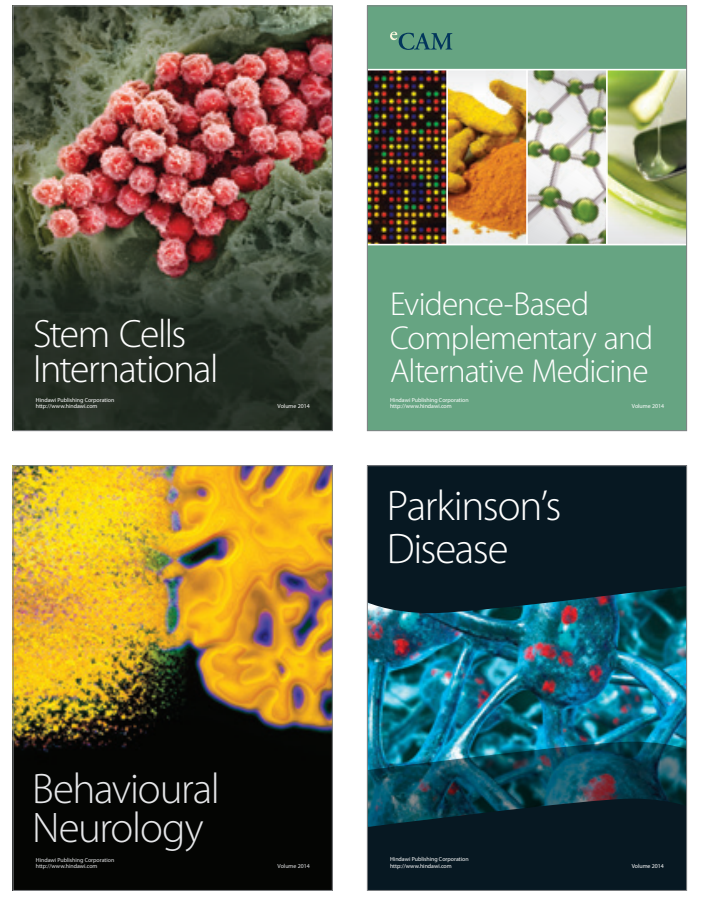
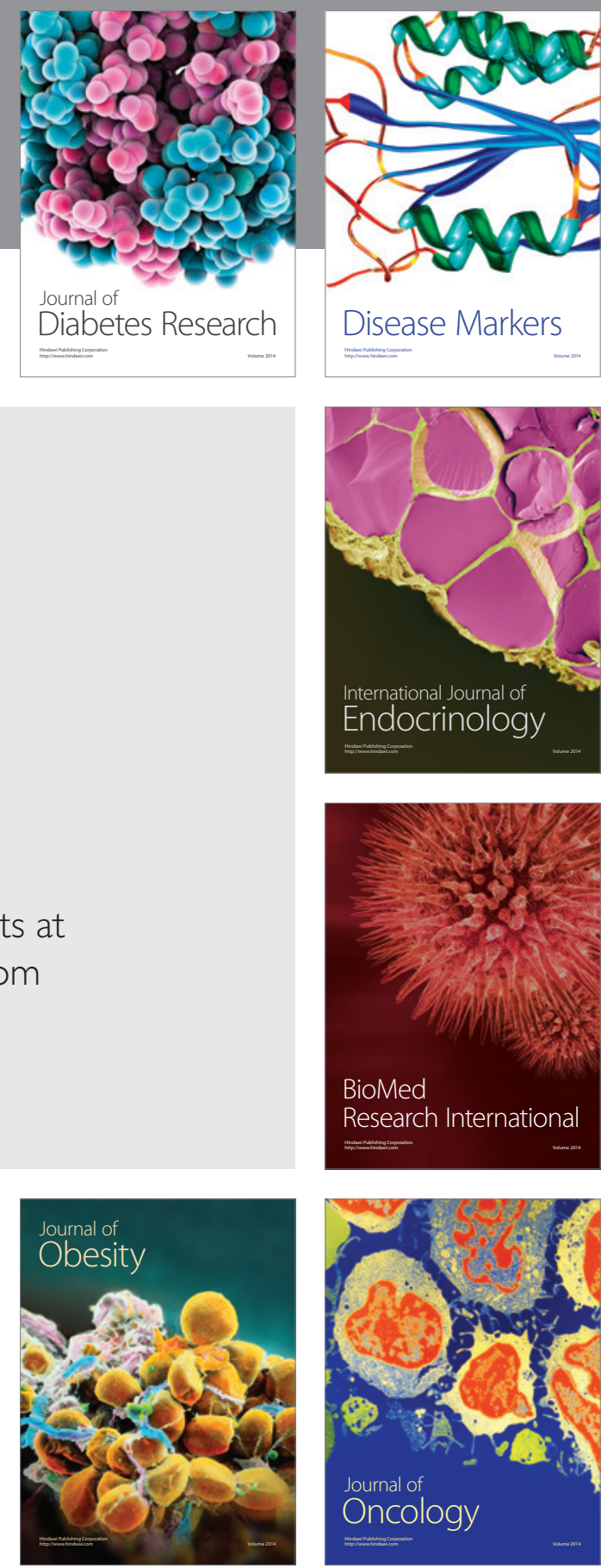

Disease Markers
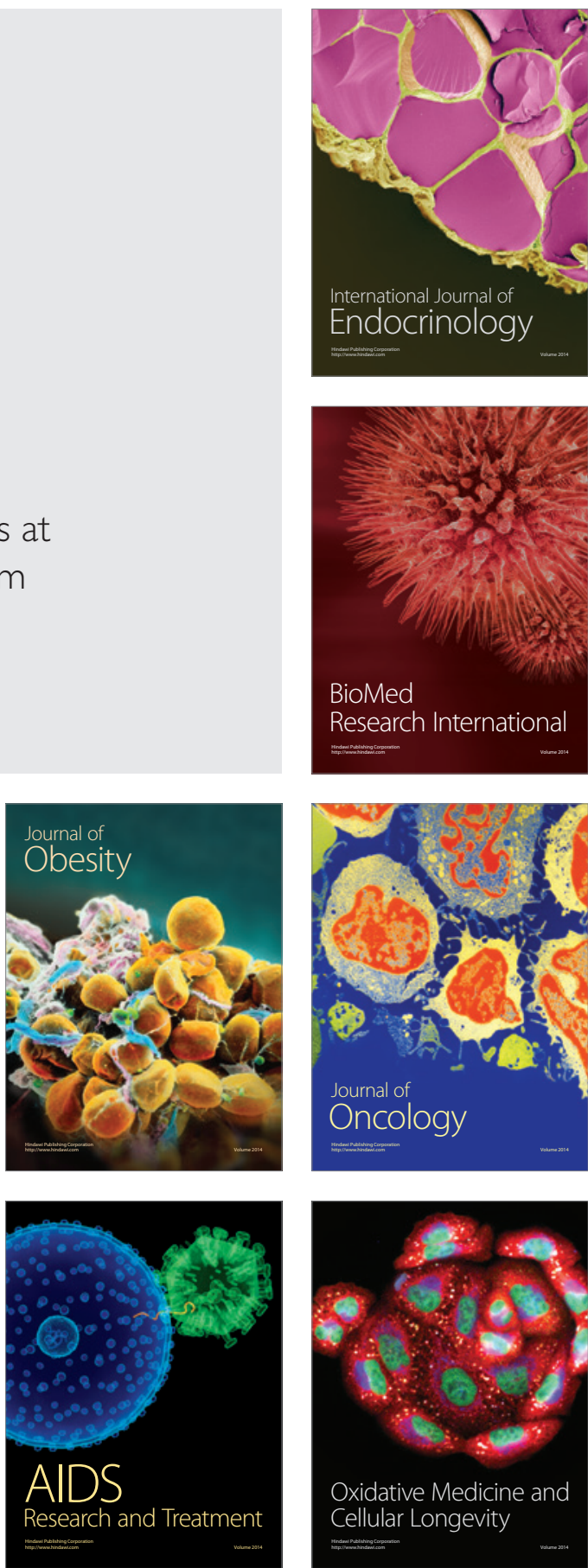